This document is confidential and is proprietary to the American Chemical Society and its authors. Do not copy or disclose without written permission. If you have received this item in error, notify the sender and delete all copies.

\title{
Electrostatic self-assembly: Understanding the significance of the solvent
}

\begin{tabular}{|r|l|}
\hline Journal: & Journal of Chemical Theory and Computation \\
\hline Manuscript ID & ct-2017-00647n.R2 \\
\hline Manuscript Type: & Article \\
\hline Date Submitted by the Author: & $\mathrm{n} / \mathrm{a}$ \\
\hline & $\begin{array}{l}\text { Brazil Lindgren, Eric; University of Nottingham School of Chemistry, } \\
\text { Derbenev, Ivan; University of Nottingham, Chemistry } \\
\text { Chan, Ho-Kei; Harbin Institute of Technology } \\
\text { Stace, Anthony; The University of Nottingham, School of Chemistry } \\
\text { Besley, Elena; University of Nottingham, Chemistry }\end{array}$ \\
\hline
\end{tabular}

SCHOLARONE ${ }^{m}$

Manuscripts 
Electrostatic self-assembly: Understanding the

\title{
significance of the solvent
}

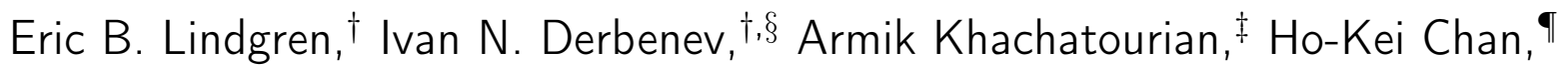 \\ Anthony J. Stace, ${ }^{\dagger}$ and Elena Besley, ${ }^{*}$ \\ $\dagger$ Department of Physical and Theoretical Chemistry, School of Chemistry, University of \\ Nottingham, University Park, Nottingham NG7 2RD, United Kingdom \\ $\ddagger$ Department of Physics and Astronomy, California State University, Los Angeles, \\ California 90032-4226, USA \\ \Shenzhen Graduate School, Harbin Institute of Technology, Shenzhen, 518055, China \\ $\S$ Troitsk Institute for Innovation and Fusion Research, Troitsk, Moscow 142190, Russia \\ E-mail: Elena.Besley@nottingham.ac.uk \\ Phone: +4401158468465
}

\begin{abstract}
The electrostatic deposition of particles has become a very effective route to the assembly of many nanoscale materials. However, fundamental limitations to the process are presented by the choice of solvent, which can either suppress or promote selfassembly depending on specific combinations of nanoparticle/surface/solvent properties. A new development in the theory of electrostatic interactions between polarizable objects provides insight into the effect a solvent can have on electrostatic self-assembly. Critical to assembly is the requirement for a minimum charge on a surface of an object, below which a solvent can suppress electrostatic attraction. Examples drawn from the literature are used to illustrate how switches in behavior are mediated by the solvent;


these in turn provide a fundamental understanding of electrostatic particle-surface interactions applicable to many areas of materials science and nanotechnology.

\section{Introduction}

The effective use of electrostatic forces in the self-assembly and fabrication of nano-materials is rapidly gaining significance in the technological development of new devices and processes. ${ }^{1}$ Examples of electrostatic self-assembly range from isolated nanoscale structures, ${ }^{2-4}$ through patterned ${ }^{5,6}$ and layered surfaces ${ }^{7}$ to macroscopic crystals consisting of $\mathrm{mm}$ diameter spherical polymer particles. $^{8}$ In many assembly processes, particularly in the fabrication of new devices, an important component in the initial stage is the self-assembled monolayer (SAM). A SAM acts as a template that enables surface characteristics to be fine-tuned for the purpose of accommodating either individual nanoparticles or multilayers in the form of layer-by-layer structures. ${ }^{9,10}$ SAMs can be either polar or non-polar, or they can be induced to attract or release protons, all of which influence the electrostatic forces experienced by nanoparticles when forming a layered surface: this electrostatic constituent is central to almost all significant developments in the field of thin film fabrication. ${ }^{11}$ If described in terms of point charges (e.g. $\mathrm{H}^{+}$and $\mathrm{O}^{-}$) located on self-assembly components, the fabrication mechanism is, at first sight, straightforward. The electrostatic force between any pair of point charges in vacuum is universally expressed by Coulomb's law. ${ }^{12}$ If the charges are immersed in a homogeneous medium or solvent of dielectric constant $k_{\text {medium }}$, the Coulomb force is attenuated by a factor equal to $k_{\text {medium }}$ as a result of a polarization charge being induced in the medium. ${ }^{12,13}$ In these circumstances, the presence of a polarizable medium does not alter the nature of the electrostatic interaction, i.e. the sign of the force remains the same. However, this simple description becomes more complicated if the interactions involve finite-sized dielectric particles and/or surfaces; ${ }^{13}$ a notable example being the counterintuitive like-charge attraction between polarizable spheres in vacuum. ${ }^{14,15}$ 
Although most self-assembly processes take place in aqueous solution ${ }^{9}$ there is a growing realization that for many of the newer materials, such as magnetic nanoparticles ${ }^{16}$ and quantum dots, ${ }^{17}$ assembly in a non-polar solvent would offer distinct advantages. ${ }^{7,18}$ In all cases, however, it is of fundamental importance to acquire a greater quantitative understanding of how interactions between charged, polarizable, finite-sized objects are influenced by the presence of a medium. Does attenuation by $k_{\text {medium }}$ still apply or does the force, and possibly the very nature of the interaction, depend critically on the dielectric constants of the various materials involved? A comprehensive answer to this question would be to the benefit of particle-surface fabrication and aggregation processes throughout many areas of materials science. To date there is only qualitative evidence for a "solvent effect" in electrostatic self-assembly; ${ }^{11,19,20}$ a quantitative analysis of such behaviour would contribute to a more rigorous evaluation of the role of the solvent.

In this paper, we demonstrate the critical nature of the solvent in determining the success of electrostatic assembly. The experimental observations ${ }^{21,22}$ chosen for discussion and numerical modeling have been selected to match most closely the framework underpinning the theory, ${ }^{14,23}$ i.e. a dielectric spherical particle interacting with a planar, dielectric surface. However, the general principles that emerge from these examples should be applicable to any electrostatic self-assembly process undertaken in the presence of a solvent. It is assumed that the solvent is not acting as or containing an electrolyte. ${ }^{\mathrm{i}}$ The intention is to focus on understanding how the solvent as a uniform dielectric medium may influence the assembly process. The effects of the presence of an electrolyte have been considered in a separate paper. ${ }^{24}$ The selected examples include a neutral particle interacting with a charged surface ${ }^{21}$ and a negatively charged particle interacting with a positively charged surface. ${ }^{22}$ Basic classical electrostatic considerations, which are only valid for point charges or non-polarizable objects, would predict a zero force in the first example and an attractive force in the latter

\footnotetext{
${ }^{\mathrm{i}}$ It is acknowledged that pure water is itself a very weak electrolyte providing screening from $\mathrm{H}^{+}$and $\mathrm{OH}^{-}$in equilibrium, but it has a Debye length that far exceeds the discussed length scales $\left(\lambda_{D} \approx 1\right.$ micron, at room temperature).
} 
case; however, our calculations reveal that the interaction can switch between repulsion and attraction, depending on the solvent and the properties of materials involved. The effect that media with different dielectric constants have on the neutral particle - point charge interaction has been illustrated previously by Barros and Luijten, ${ }^{25}$ and they also present a numerical solution to the case where the particle carries a charge.

The numerical results presented here are derived from analytical theory ${ }^{14,23}$ capable of explaining many of experimental outcomes likely to be encountered during the electrostatic fabrication of particle - particle and particle - surface assemblies. Previous attempts ${ }^{26}$ to calculate the electrostatic force in a two-particle system include the application of an accurate re-expansion method ${ }^{27,28}$ proposed by Washizu and co-workers, however convergence of the re-expansion method gets generally poor if the ratio of the radii of the spheres is large and if the particles are closely spaced. To address a potentially similar covergence problem, we apply an efficient numerical discretisation method proposed by Lindgren and co-workers ${ }^{29}$ to an infinite sum of Legendre polynomials representing the electrostatic force in the mathematical solutions ${ }^{14,23}$ used in this study, which allows us to include as many terms in the expansion as needed and achieve very effectively computationally the desired even very tight - convergence in the quantitative estimation of the electrostatic force. This numerical capability is particularly important in the cases where interacting particles have high values of the dielectric constant, are separated by small distances, and/or largely differ in size. Additionally, our solution, as indeed any method based on a multipole expansion of the electrostatic force, provides a meaningful physical insight into the interaction problem, for example by presenting an exact surface charge distribution on interacting particles, which varies with separation distance. Lindell and co-workers ${ }^{30}$ generalized the classical image charge solution to a system composed by two dielectric spheres suspended in a medium. This solution also suffers from the convergence problem, intrinsic to all image charge methods, as the separation between interacting particles becomes small. 


\section{Electrostatic model}

The new insight into how the medium influences electrostatic interactions between charged dielectric materials builds on work published previously, ${ }^{14,23}$ where analytical expressions have been given for the electrostatic force between charged, dielectric sphere - sphere and charged, dielectric sphere - planar surface systems in vacuum. In these electrostatic models, the mutual effect of charge is obtained from Gauss's law, which couples uniquely the electrostatic potential with the distribution and magnitude of electric charge on the surfaces of the interacting objects. The accumulated surface charge is integrated to obtain an analytical expression for the electrostatic force acting on the interacting objects at arbitrary separation. The obtained result is a simple series expression for the force that can be efficiently generalized for studying interactions in solutions.

In this section, we introduce a uniform homogeneous dielectric medium into the Maxwell formalism. In the models, ${ }^{14,23}$ the free charge distributed over the surfaces of the interacting objects defines an interface discontinuity in the normal component of the electric displacement field, such that: ${ }^{31}$

$$
\sigma_{\text {free }}=\left(\mathbf{D}_{\text {medium }} \cdot \hat{\mathbf{n}}\right)-\left(\mathbf{D}_{\text {object }} \cdot \hat{\mathbf{n}}\right)
$$

where $\sigma_{\text {free }}$ is the free charge density at the boundary between the object and the medium, $\mathbf{D}_{\text {medium }}$ and $\mathbf{D}_{\text {object }}$ are, respectively, electric displacement vectors in the medium and in the object, and $\hat{\mathbf{n}}$ is a unit vector directed towards the medium. In an isotropic and uniform medium, the displacement vector is generally related to the electric field via the permittivity, $\varepsilon$, as $\mathbf{D}=\varepsilon \mathbf{E}$. Therefore, Equation 1 can be re-written as:

$$
\frac{1}{\varepsilon_{0}} \frac{\sigma_{\text {free }}}{k_{\text {medium }}}=\left(\mathbf{E}_{\text {medium }} \cdot \hat{\mathbf{n}}\right)-\frac{k_{\text {object }}}{k_{\text {medium }}}\left(\mathbf{E}_{\text {object }} \cdot \hat{\mathbf{n}}\right),
$$

where $k_{\text {object }}=\varepsilon_{\text {object }} / \varepsilon_{0}$ and $k_{\text {medium }}=\varepsilon_{\text {medium }} / \varepsilon_{0}, \mathbf{E}_{\text {object }}$ is the electric field generated inside the object, $\mathbf{E}_{\text {medium }}$ is the electric field generated in the medium, and $\varepsilon_{\text {object }}, \varepsilon_{\text {medium }}$ 
and $\varepsilon_{0}$ are the permittivity of the object, the medium and free space, respectively. The total surface charge density, $\sigma_{\text {total }}$, is the sum of the free and bound surface charge densities. The free charge has external origin and arises from some form of ionization process. In contrast, the bound charge is induced at an interface by the presence of an external electric field generated by an adjacent charged object. The total charge distribution generates the resultant electric field both inside and outside the interacting objects so that it defines an interface discontinuity in the normal component of the electric field, such that

$$
\frac{1}{\varepsilon_{0}} \sigma_{\text {total }}=\left(\mathbf{E}_{\text {medium }} \cdot \hat{\mathbf{n}}\right)-\left(\mathbf{E}_{\text {object }} \cdot \hat{\mathbf{n}}\right)
$$

Following Maxwell's derivation ${ }^{32}$ of the electrostatic force acting on a object with charge $Q_{i}$ from a object with charge $Q_{j}, i=1,2, j=3-i$ :

$$
\begin{aligned}
\mathbf{F}_{i j} & =K \int d Q_{i}\left(\mathbf{x}_{i}\right) \int d Q_{j}\left(\mathbf{x}_{j}\right) \frac{\mathbf{x}_{i}-\mathbf{x}_{j}}{\left|\mathbf{x}_{i}-\mathbf{x}_{j}\right|^{3}} \\
& =\int_{V_{i}} \mathbf{E}_{j} \rho_{i} d^{3} \mathbf{r}_{i}=\varepsilon_{0} \int_{V_{i}} \mathbf{E}_{j}\left(\nabla \cdot \mathbf{E}_{i}\right) d^{3} \mathbf{r}_{i},
\end{aligned}
$$

where

$$
\mathbf{E}_{j}=K \int d Q_{j} \frac{\mathbf{x}_{i}-\mathbf{x}_{j}}{\left|\mathbf{x}_{i}-\mathbf{x}_{j}\right|^{3}}=K \int_{V_{j}} \frac{\rho_{j} d^{3} \mathbf{r}_{j}}{r_{i j}^{3}} \mathbf{r}_{i j}
$$

is the electric field generated by the second object, $\mathbf{r}_{i j}=\mathbf{x}_{i}-\mathbf{x}_{j}, \rho_{i}$ is the volume charge density $\left(d Q_{i}=\rho_{i} d^{3} \mathbf{r}_{i}\right)$, and $\nabla \cdot \mathbf{E}_{i}=\rho_{i} / \varepsilon_{0}$ in accordance with Gauss's law. The total field and volume charge density are $\mathbf{E}=\mathbf{E}_{1}+\mathbf{E}_{2}$ and $\rho=\rho_{1}+\rho_{2}$, respectively. The volume $V_{i}$ and the closed surface $S_{i}$ contain the object $Q_{i}$ only, and they are in close proximity to its boundary. Therefore, within $S_{i}, \rho=\rho_{i}$ or $\nabla \cdot \mathbf{E}=\nabla \cdot \mathbf{E}_{i}$. Since the object $Q_{i}$ cannot act on itself the integral $\varepsilon_{0} \int_{V_{i}} \mathbf{E}_{i}\left(\nabla \cdot \mathbf{E}_{i}\right) d^{3} \mathbf{r}_{i}=0$. This allows the force to be rewritten as 


$$
\mathbf{F}_{i j}=\varepsilon_{0} \int_{V_{i}} \mathbf{E}(\nabla \cdot \mathbf{E}) d^{3} \mathbf{r}
$$

The integrated product of the electric field vector on its divergence can be represented as the divergence of the tensor:

$$
\varepsilon_{0} \mathbf{E}(\nabla \cdot \mathbf{E})=(\nabla \cdot \mathbf{T})
$$

where $T_{i j} \equiv \varepsilon_{0}\left(E_{i} E_{j}-\frac{1}{2} \delta_{i j} E^{2}\right)$ is the Maxwell stress tensor in vacuum. Then

$$
\mathbf{F}_{i j}=\int_{V_{i}}(\nabla \cdot \mathbf{T}) d^{3} \mathbf{r}=\oint_{S_{i}}(\mathbf{T} \cdot \mathbf{n}) d S=\varepsilon_{0} \oint\left[\mathbf{E}(\mathbf{n} \cdot \mathbf{E})-\frac{1}{2}(\mathbf{E})^{2} \mathbf{n}\right] d S
$$

Finally, the electrostatic force in a non-deformable isotropic dielectric medium is: ${ }^{33}$

$$
\mathbf{F}_{\text {medium }}=k_{\text {medium }} \varepsilon_{0} \oint\left[\mathbf{E}(\mathbf{n} \cdot \mathbf{E})-\frac{1}{2}(\mathbf{E})^{2} \mathbf{n}\right] d S=k_{\text {medium }} \mathbf{F}_{i j} .
$$

In order to investigate the components of the electrostatic force $\mathbf{F}_{i j}$, representing contributions from the medium and objects separately, an alternative 'four-layer' model has been developed, which is described in detail in Appendix A. This alternative solution gives the same value for the electrostatic force as Equation 8 showing a complete quantitative agreement between these two approaches.

\section{Results and discussion}

Two coupled phenomena that define the nature of the electrostatic interaction in a medium are an interfacial redistribution of polarization charge and a reduction in the force due to the presence of a medium. The critical parameter for the interaction is the ratio between the permittivity of the interacting object and the medium, $k_{\text {object }} / k_{\text {medium }}$. If $k_{\text {object }} / k_{\text {medium }} \approx 1$, polarization effects become negligible and the presence of the medium simply decreases the 
magnitude of the force. In contrast, when $k_{\text {object }} / k_{\text {medium }} \ll 1$ or $k_{\text {object }} / k_{\text {medium }} \gg 1$, the charge induced at the interface between the medium and the interacting objects may promote a marked change in the magnitude of the force. Also, the nature of the electrostatic force may change switching from attractive to repulsive and vice versa, as indicated by a change of sign in the force. This effect was qualitatively described by Israelachvili ${ }^{13}$ using the simple dipole approximation, and also predicted by $\mathrm{Wu}$ and co-workers ${ }^{34}$ for neutral and charged Janus particles with various permittivities, immersed in symmetric and asymmetric electrolytes.

A straightforward example of how the properties of a solvent could either facilitate or suppress electrostatic fabrication, can be seen from experiments ${ }^{21}$ involving the interaction of neutral spherical alumina particles (approximately $50 \mathrm{~nm}$-diameter and dielectric constant $\left.k_{\text {particle }}=9.9\right)$ with a charged nano-diamond surface of dielectric constant $k_{\text {surface }}=5.3$. The expectation is that a neutral particle will be attracted to a surface with a given charge density of $\sigma_{\text {surface }}=+1 \mathrm{e} \cdot \mathrm{nm}^{-2}$, and that is exactly what the experiments show if the particles are immersed in a liquid medium of dielectric constant $k_{\text {medium }}=1.86$ (insulating fluorocarbon solution, fluorinert FC-90). Figure 1a reinforces this observation by showing that the computed electrostatic force is attractive at all separation distances up to touching point (a negative force denotes attraction). However, further calculations show that the nature of the interaction can switch markedly from being attractive to repulsive if a solvent with a dielectric constant, $k_{\text {medium }}=20$, for example, acetone, were to be used instead (Figure 1b).

This transition from attractive to repulsive electrostatic behaviour occurs at $k_{\text {medium }}=$ $k_{\text {particle }}=9.9$, where the lack of a dielectric discontinuity at the interface between the particle and the medium implies an absence of polarization charge resulting in the elimination of any electrostatic interaction between the particle and the surface. At $k_{\text {medium }}=1.86$, where the medium is less polarizable than both the alumina particle and the nano-diamond surface, the positively charged, planar surface can induce an effective dipole on the neutral particle, which as shown in Figure 2a, points away from the surface. Since the negative component 

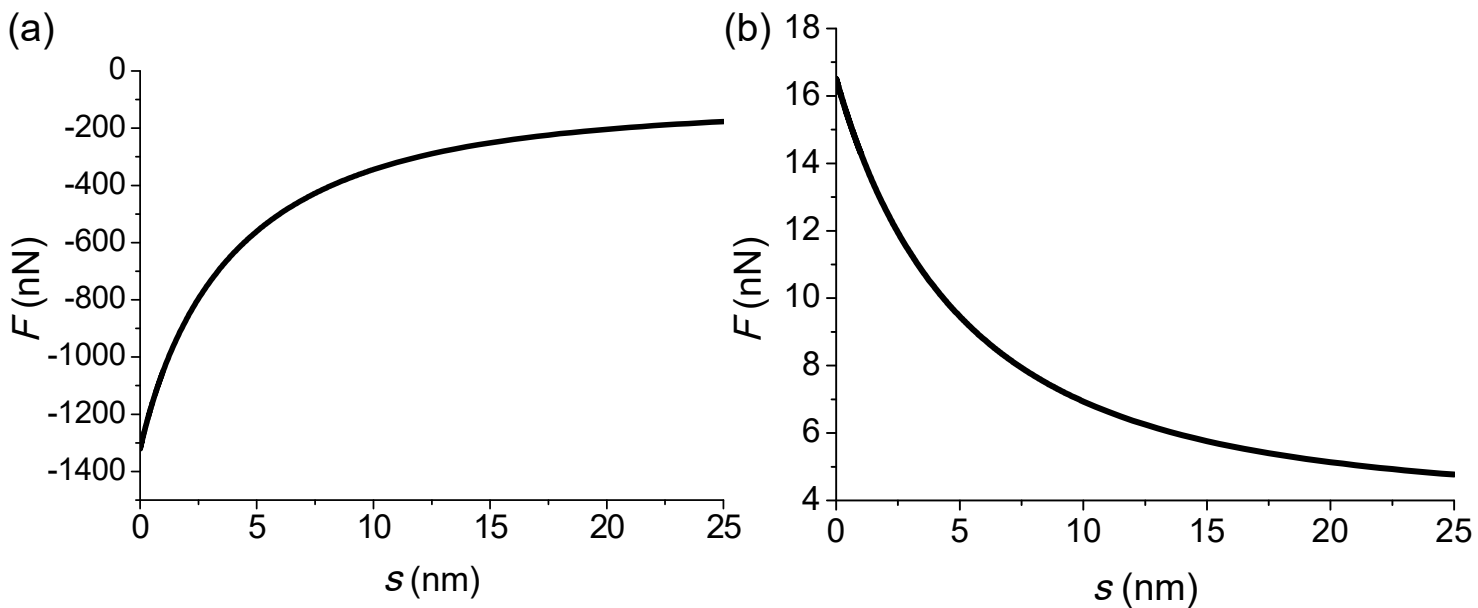

Figure 1: Electrostatic force $(\mathrm{nN})$ as a function of the surface-to-surface separation between a neutral alumina spherical particle and a charged nano-diamond surface immersed in a medium with dielectric constant $k_{\text {medium }}=1.86$ (a) and $k_{\text {medium }}=20(\mathrm{~b})$. The particle has a radius of $25 \mathrm{~nm}$ and a dielectric constant $k_{\text {particle }}=9.9$. The surface charge density of the planar surface is $\sigma_{\text {surface }}=+1 \mathrm{e} \cdot \mathrm{nm}^{-2}$, and the dielectric constant is $k_{\text {surface }}=5.3$.

of the dipole is adjacent to the positively charged surface, the overall interaction between the particle and the surface is dominated by an attractive electrostatic force. However, if a solvent with a higher dielectric constant is chosen, i.e. $k_{\text {medium }}=20$, the medium is now more polarizable than either the particle or the surface and, as shown in Figure 2b, there is a corresponding switch in the direction of the induced dipole. The result is a net repulsive interaction between the particle and the surface, which would not be predicted from a simple application of Coulomb's law. Note also that, although a charge density of $+1 \mathrm{e} \cdot \mathrm{nm}^{-2}$ has been imposed on the surface, the effective charge experienced by the neutral particle is severely depleted in the presence of a high dielectric solvent. The observed repulsive interaction between a neutral particle and a charged planar surface will persist with high-dielectric solvents, such as water $\left(k_{\text {medium }}=80\right)$ that are increasingly more polarizable than the components offered for electrostatic self-assembly. For a neutral - charged pair of objects, further calculations show that neither changing the sign of the charge on the planar surface nor increasing the magnitude of the charge on the surface can change these circumstances.

As a second illustration of the importance of the solvent in influencing fabrication pro- 
(a)

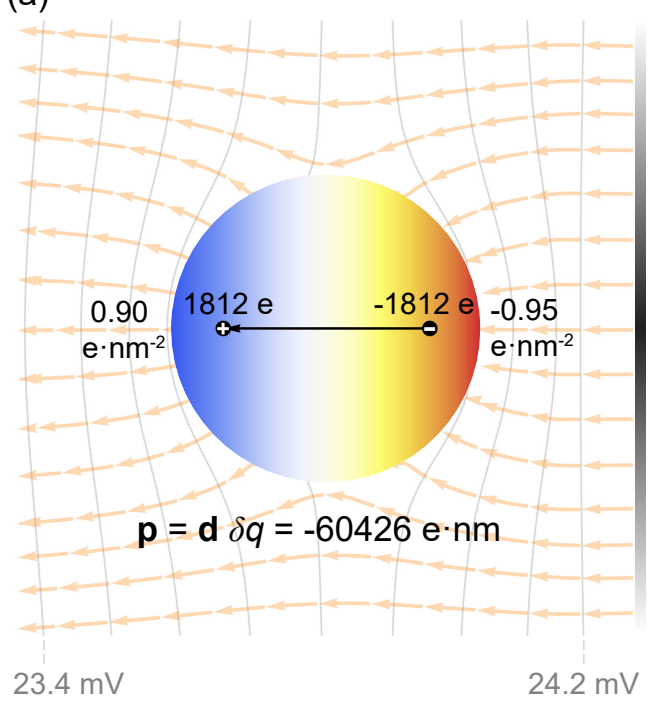

(b)

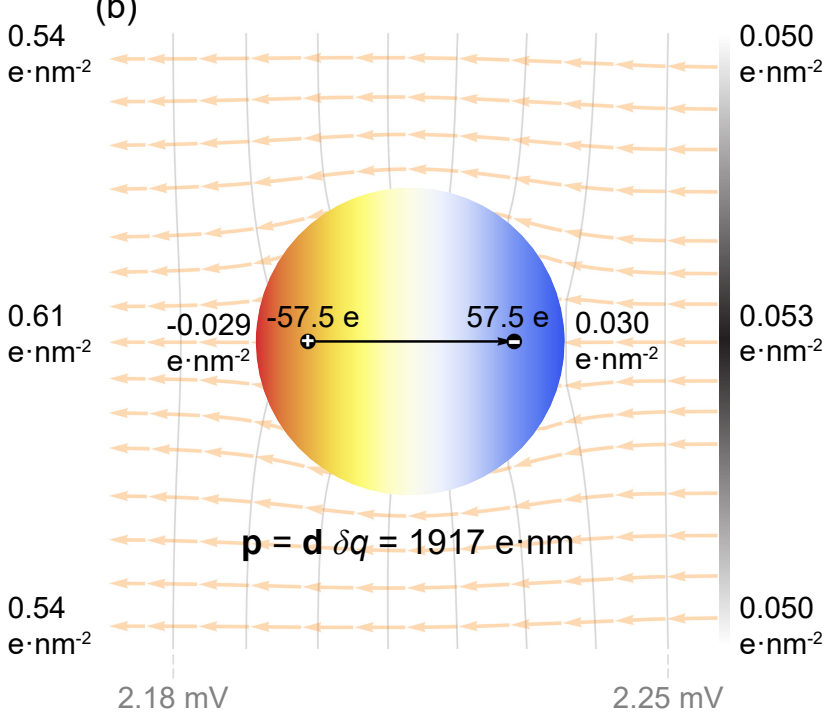

Figure 2: Schematic illustration of the electrostatic interaction corresponding to the case presented in Figure 1, showing equipotential lines, the electric field, the effective dipole moment in the neutral alumina particle (see Appendix B), values of the effective surface charge density at selected points, and values of the electric potential at selected points away from the surface. Note the switch in the direction of the effective dipole moment when the dielectric constant of the solvent changes from $k_{\text {medium }}=1.86$ (a) to $k_{\text {medium }}=20$ (b).

cesses, the assembly of thin films from polyoxometalate (POM) nanoclusters has been examined. Nanostructured materials containing POMs are increasingly seen as an attractive route to the development of functional materials and devices; ${ }^{22,35}$ however, to achieve these objectives, it is necessary to develop a strategy for creating coherent thin-film structures. Most POMs are soluble in water; ${ }^{36}$ but it is widely recognized that from such a polar medium it is not possible to fabricate layers without first creating a charged substrate. ${ }^{22}$ The following calculations have been designed to model deposition of the Eu-POM onto a layer of positively charged macromolecules in the form of a planar substrate. ${ }^{22}$ Liu et al. have subsequently shown that this particular combination of materials can form the base of an electrochromic device. ${ }^{35}$ The Eu-POM is an anion, $\left[\mathrm{Eu}\left(\mathrm{H}_{2} \mathrm{O}\right) \mathrm{P}_{5} \mathrm{~W}_{30} \mathrm{O}_{110}\right]^{12-}$, and a polyelectrolyte layer residing on a quartz or silicon substrate provides the positive charge. The charge density associated with the substrate is unknown, and it is this quantity that is explored here in terms of the minimum density required to promote the surface deposition of POMs in a solvent with a given dielectric constant. Although the presence of an electrolyte $(\mathrm{NaCl})$ does 
improve surface coverage, it has been shown that fabrication can proceed in the absence of sodium chloride. ${ }^{22}$

Table 1 shows the calculated electrostatic force between $\left[\mathrm{Eu}\left(\mathrm{H}_{2} \mathrm{O}\right) \mathrm{P}_{5} \mathrm{~W}_{30} \mathrm{O}_{110}\right]^{12-}$ and a uniform polyelectrolyte layer as a function of both the charge density on the substrate and the dielectric constant of the solvent in which the Eu-POM is suspended (a negative force denotes an attractive interaction). As can be seen, at very low surface charge densities, the two interacting objects are only attracted to one another in vacuum $\left(k_{\text {medium }}=1\right)$ and in all other solvents a layered structure would fail to form. The reason for this failure is related to the observations accompanying Figure 1, in that for constituents with the dielectric constants $k_{\mathrm{POM}}=10$ and $k_{\text {surface }}=5$ in the presence of a solvent where $k_{\text {medium }} \geq 10$, there will be a weakly repulsive electrostatic force preventing assembly. However, as Table 1 shows, a gradual increase in surface charge density reveals the existence of a critical density, $\sigma_{\text {critical }}=0.1 \mathrm{e} \cdot \mathrm{nm}^{-2}$, beyond which layer formation is assured for all solvents including water.

To demonstrate the consequence of introducing a solvent in terms of changes that take place in the distribution of charge, Figure 3 gives a graphical illustration of an example taken from Table 1. For a net charge of -12 e on the POM and a planar surface charge density of $+0.05 \mathrm{e} \cdot \mathrm{nm}^{-2}$, changes in density as the two interacting objects approach one another have been calculated for vacuum (Figure 3a) and for a solvent with a dielectric constant of 20 (Figure 3b). For the vacuum case, Figure 3a also shows how charge density on the surface changes from $\sigma_{\text {surface }}=+0.05 \mathrm{e} \cdot \mathrm{nm}^{-2}$, at a radial distance of $10 \mathrm{~nm}$ from the POM, to $\sigma_{\text {surface }} \approx+0.6 \mathrm{e} \cdot \mathrm{nm}^{-2}$ immediately below the POM. A comparable change in negative charge density is seen on the POM, and the net result is that oppositely charged bodies attract. With the introduction of a solvent, the transition in behaviour is dramatic. The POM retains a negative charge, but the presence of the surface has minimal effect on how it is distributed. In contrast, the planar surface is, as expected, positively charged at a radial distance of $10 \mathrm{~nm}$ from the POM, but as Figure 3b shows, in close proximity to the POM, 
Table 1: The electrostatic force (in $\mathrm{pN}$ ) calculated for the experimental case ${ }^{22,35}$ of an interaction between a Eu-POM, $\left[\mathrm{Eu}\left(\mathrm{H}_{2} \mathrm{O}\right) \mathrm{P}_{5} \mathrm{~W}_{30} \mathrm{O}_{110}\right]^{12-}$, and an oppositely charged planar surface at the surface-to-surface separation distance of $1 \mathrm{~nm}$. Calculations have been undertaken using different combinations of charge density on the planar surface $\left(\sigma_{\text {surface }}\right)$ and dielectric constant of the medium $\left(k_{\text {medium }}\right)$. The POM particle has a radius of 0.5 $\mathrm{nm}$ and an estimated dielectric constant of $k_{\mathrm{POM}=10^{\dagger}}$. The planar surface has a coating of poly(ethylenimine)/poly(styrenesulfonate)/poly(allylamine hydrochloride) with an estimated dielectric constant of $k_{\text {surface }}=5 .{ }^{37}$ The charge density on the planar surface is in units of $\mathrm{e} \cdot \mathrm{nm}^{-2}$.

\begin{tabular}{c|ccccccc}
$k_{\text {medium }} \downarrow / \sigma_{\text {surface }} \rightarrow$ & 0.001 & 0.005 & 0.01 & 0.05 & 0.1 & 0.5 & 1 \\
\hline 1 & -2510.4 & -2649.6 & -2823.7 & -4216.2 & -5957.1 & -19889.0 & -37317.1 \\
10 & 118.8 & 105.0 & 87.7 & -50.2 & -222.7 & -1602.2 & -3326.6 \\
20 & 108.4 & 101.5 & 92.9 & 23.8 & -62.7 & -753.9 & -1617.9 \\
30 & 86.4 & 81.8 & 76.0 & 29.8 & -27.8 & -489.4 & -1066.2 \\
40 & 70.7 & 67.2 & 62.9 & 28.2 & -15.1 & -361.6 & -794.6 \\
50 & 59.6 & 56.8 & 53.3 & 25.6 & -9.1 & -286.5 & -633.1 \\
60 & 51.4 & 49.1 & 46.2 & 23.0 & -5.9 & -237.1 & -526.1 \\
70 & 45.1 & 43.2 & 40.7 & 20.8 & -4.0 & -202.3 & -450.1 \\
80 & 40.2 & 38.5 & 36.3 & 19.0 & -2.7 & -176.3 & -393.2
\end{tabular}

†There do not appear to be any reliable data on the dielectric constants of POMs. A value of 10 is based on values for metal oxides., ${ }^{38}$ and metal-containing nanoparticles. ${ }^{39}$

the charge on the surface changes sign to become $\sigma_{\text {surface }} \approx-0.25 \mathrm{e} \cdot \mathrm{nm}^{-2}$ at the point of closest contact. The net result now is that the two interacting objects repel one another. As demonstrated in Figure 2, polarization of the solvent leads to a dramatic attenuation of charge density; however, in that example, it was the neutral particle that switched density, whereas in Figure 3, it is the charge on the planar surface that is most influenced by the choice of solvent.

There are several examples in the literature of deposition processes where there is evidence of a critical charge density being required for the growth of layers. ${ }^{10,40}$ The exact numbers in Table 1 are specific to the model system being studied; however, it is instructive to see what the values equate to in molecular terms: given that a Eu-POM has a diameter of approximately $1 \mathrm{~nm}$, which would give it a projected surface coverage of $3.14 \mathrm{~nm}^{2}$, the required critical surface charge density is less than one proton per POM. If, however, the attractive force needs to be greater than the minimum, then at $\sigma_{\text {surface }}=+0.5 \mathrm{e} \cdot \mathrm{nm}^{-2}$, for 
(a)

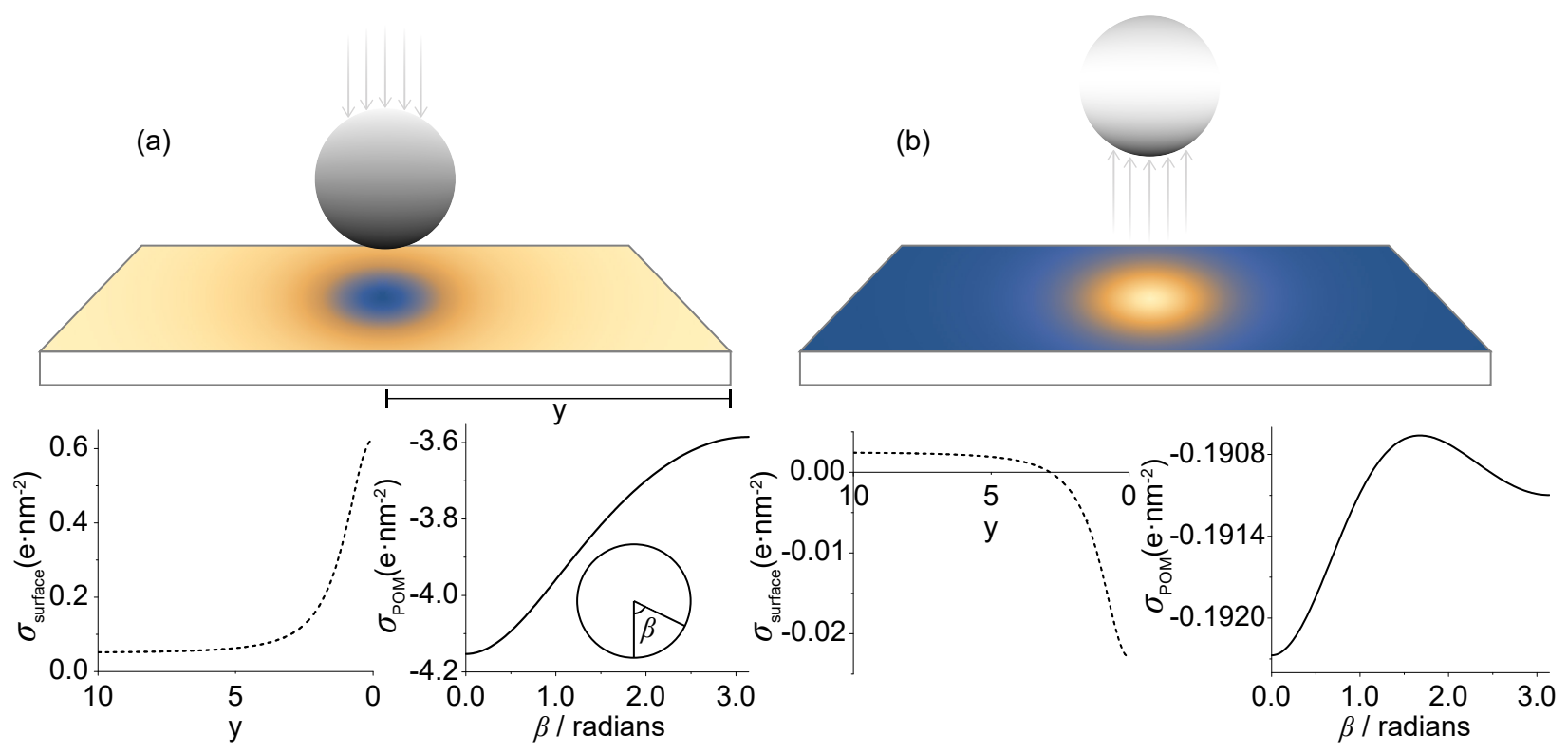

Figure 3: Schematic illustration of the POM - surface interaction. In (a) the medium/solvent has a dielectric constant of 1 (vacuum) and in (b) the medium/solvent has a dielectric constant of 20 . The planar surface has a surface charge density of $+0.05 \mathrm{e} \cdot \mathrm{nm}^{-2}$ and the POM particle has a net charge of -12 e. The total charge density on the planar surface, $\sigma_{\text {surface }}$ (dashed curve) and on the POM particle, $\sigma_{\mathrm{POM}}$ (solid curve) are also shown for each interaction case: for $\sigma_{\text {surface }}$, the $x$-axis denotes the radial position of charge $(y)$, with $y=0$ indicating the center of POM projected on the surface; for $\sigma_{\mathrm{POM}}$, the $x$-axis denotes the polar angle $(\beta)$, where $\beta=0$ represents the nearest point to the plane. The colour shades on both objects reflect qualitatively the calculated charge distribution, however the colour scale is different in each example.

example, the surface coverage would need to be closer to two protons per POM. Some of the experimental evidence for a critical charge density in layer-by-layer assembly is complicated by the need to invert surface charge in order to accommodate subsequent layers ${ }^{41}$ however, there is supporting evidence for such an effect when, under aqueous conditions, polystyrene nanoparticles with varying degrees of negative charge are adsorbed onto the surface of a positively charged polymer film. ${ }^{42}$

With a view to understanding at a general level the conditions required for electrostatic self-assembly, Figure 4 summarizes a series of extensive calculations that explore the consequences of charged particles interacting in solvents with a range of dielectric constants. The calculations cover interactions between both opposite- and like-charged particles, and 
size ratios which span from particles of equal size through to differences in size that are almost representative of a particle - planar surface interaction (see Appendix C). In each of Figures 4a-4f, regions of repulsion between the two particles are shown in purple. Given that each particle has a dielectric constant of 10 , the most dramatic changes take place as the dielectric constant of the solvent shifts from 5 through to 15 . At $k_{\text {medium }}=5$, all interactions where the particles are oppositely charged are attractive, as are a significant fraction of those between like-charged particles, but under two quite distinct sets of conditions. Attraction between like-charged particles occurs when there are either large differences in particle size or when the particles are comparable in size, but carry significantly different amounts of charge; in both cases the attraction arises from an enhanced polarization of surface charge. As $k_{\text {medium }}$ switches from a value of 9 to one of 11, there is a dramatic change in the nature of the particle-particle interaction; now each particle becomes actively engaged in polarizing the solvent rather than the adjacent particle, and surface charge at the particle - solvent interface can cause net repulsion, even when the particles carry opposite charges (see Figure 3b). As the dielectric constant of the solvent increases in magnitude, the region of attraction between oppositely charged particles diminishes markedly, but remains predominantly dependent on not so large differences in charge density between the interacting particles. In effect, there is a critical charge density, below which there is no attraction cf. Table 1.

There are important differences between the two examples of self-assembly discussed above. In the first case, any attractive interaction between the charged nano-diamond surface and the neutral alumina particle is due entirely to polarization effects, and these are readily suppressed when the dielectric constant of the medium exceeds that of the interacting constituents. In contrast, the second example represents a case where oppositely charged constituents should experience an interaction, which Coulomb's law would describe as being attractive under all circumstances (vacuum and solvent). However, that is clearly not the case, and the calculations reveal the existence of a critical charge density that is required to be present on a substrate in order to overcome polarization effects in a medium that may 

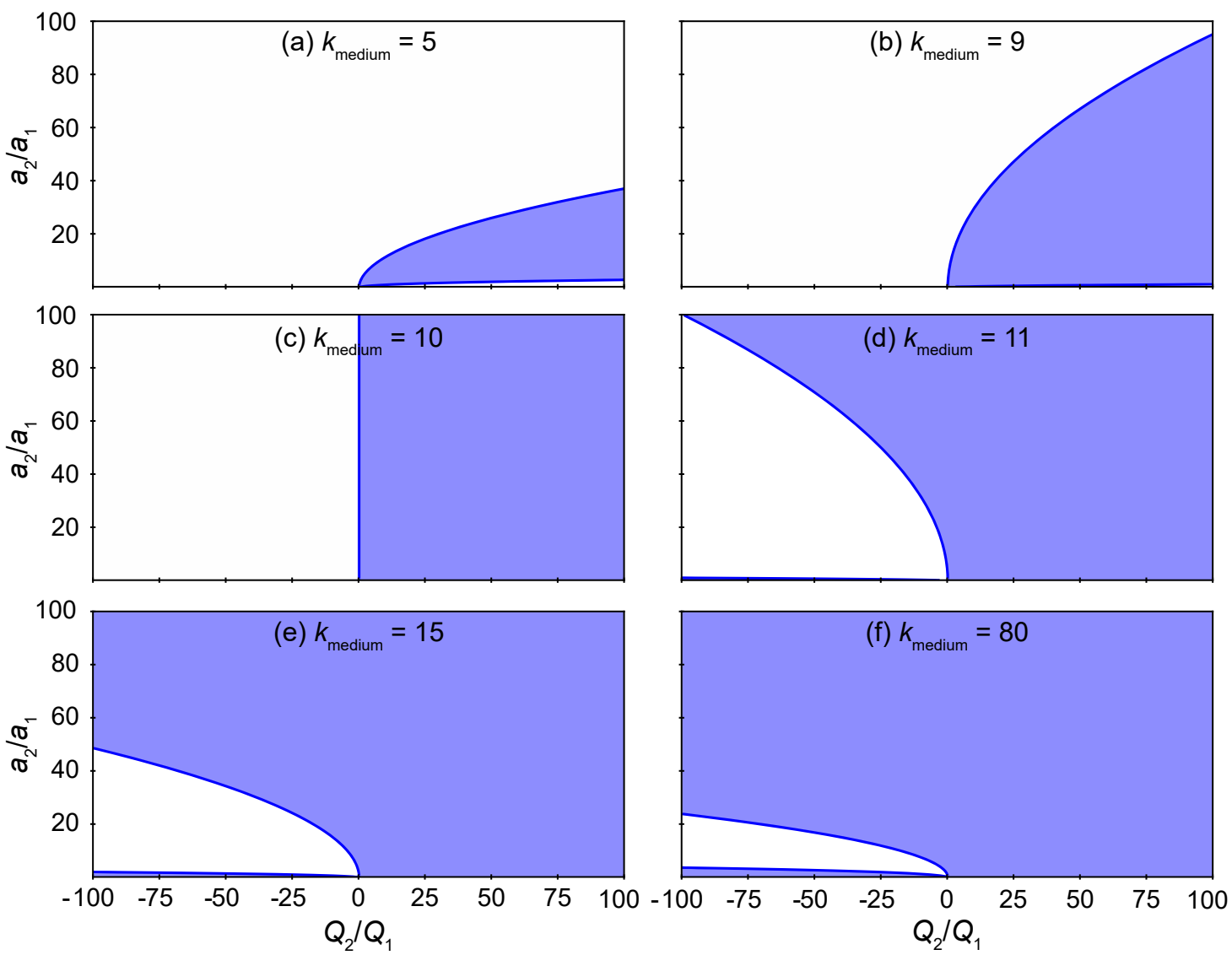

Figure 4: Electrostatic maps showing regions of repulsive (light purple) and attractive (white) interactions. The electrostatic force has been calculated as a function of the charge ratio, $Q_{2} / Q_{1}$ and the radius ratio, $a_{2} / a_{1}$, with the radius and charge of particle 1 fixed at $a_{1}=1$ and $Q_{1}=1$. The dielectric constants of the particles are $k_{1}=k_{2}=10$ and the calculations have been undertaken at a fixed separation distance of $s=0.1 a_{1}$.

serve to suppress any attraction.

\section{Conclusion}

A consistent message to emerge from the calculations presented in this work is that electrostatic self-assembly is more likely to proceed if undertaken in a solvent with a low dielectric constant. ${ }^{7}$ Although such conditions make it more difficult for particles and surfaces to acquire charge, the calculations show that assembly can proceed at significantly lower levels of charge than are typical for an aqueous medium. The absence of strong screening also means that low charge levels can be effective over large distances, which again should be important 
for self-assembly.

\section{Appendix A: An alternative 'four-layer' model}

An alternative approach to calculating the force between two spheres in a medium is a 'fourlayer' formalism, based on the bispherical coordinate system (Figure 5). In this alternative model (see Figure 6), two spheres with dielectric constants $k_{1}$ and $k_{5}$, radii $a_{1}$ and $a_{4}$, and free constant surface charges, $\sigma_{1}$ and $\sigma_{4}$, respectively, are each surrounded by a thin layer of spherical vacuum, $k_{2}=k_{4}=1$, embedded in a polarizable medium whose dielectric constant is $k_{3}$. The thickness of the vacuum layer surrounding each sphere is assumed to approach zero. This construction allows an unambiguous separation of the spheres from the surrounding medium. The electrostatic force acting on sphere 1 then corresponds to the sum of the forces between (i) the charge residing on the surface of the second sphere with the charge residing on the surface of sphere 1; (ii) the charge residing on the surface of the second sphere with the polarization charge (originated from the medium) residing a distance $a_{2}$ from the center of the sphere 1 , (iii) the polarization charge (originated from the medium) residing a distance $a_{3}$ from the center of the second sphere with the charge residing on the surface of sphere 1, and (iv) the polarization charge (originated from the medium) residing a distance $a_{3}$ from the center of the second sphere with the polarization charge (originated from the medium) residing a distance $a_{2}$ from the center of the sphere 1 . The resultant electrostatic force can be written as:

$$
\mathbf{F}_{i j} \equiv \mathbf{F}_{12 o n 34}=K \sum_{i=1}^{2} \sum_{j=3}^{4} \int d Q_{i} \int \frac{d Q_{j}}{r_{i j}^{3}} \mathbf{r}_{i j}=K \int d Q_{12} \int \frac{d Q_{34}}{r_{14}^{3}} \mathbf{r}_{14}
$$

where $d Q_{i j}=d Q_{i}+d Q_{j}$. 


$$
\begin{aligned}
\mathbf{F}_{12 o n 34}= & K \int d Q_{1,2} \int \frac{d Q_{3,4}}{r_{1,4}^{3}} \mathbf{r}_{1,4}=K \int d Q_{1,2}\left(\mathbf{x}_{1}\right) \int d Q_{3,4}\left(\mathbf{x}_{2}\right) \frac{\mathbf{x}_{1}-\mathbf{x}_{2}}{\left|\mathbf{x}_{1}-\mathbf{x}_{2}\right|^{3}} \\
= & \frac{1}{K} \sum_{n=0}^{\infty}\left[\Phi_{3, n} e^{-\left(n+\frac{1}{2}\right)\left(\eta_{1}-\eta_{3}\right)}+\Phi_{4, n} e^{-\left(n+\frac{1}{2}\right)\left(\eta_{1}-\eta_{4}\right)}\right] \\
& \times\left[e^{\eta_{1}} \frac{n}{2}\left(\Phi_{1, n-1}+\Phi_{2, n-1}\right)-\left(n+\frac{1}{2}\right)\left(\Phi_{1, n}+\Phi_{2, n}\right)\right. \\
& \left.+e^{-\eta_{1}} \frac{n+1}{2}\left(\Phi_{1, n+1}+\Phi_{2, n+1}\right)\right] .
\end{aligned}
$$

(a)

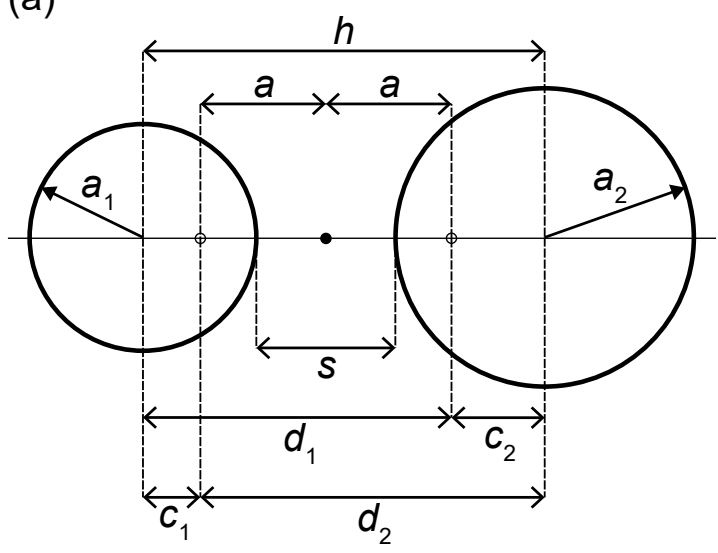

(b)

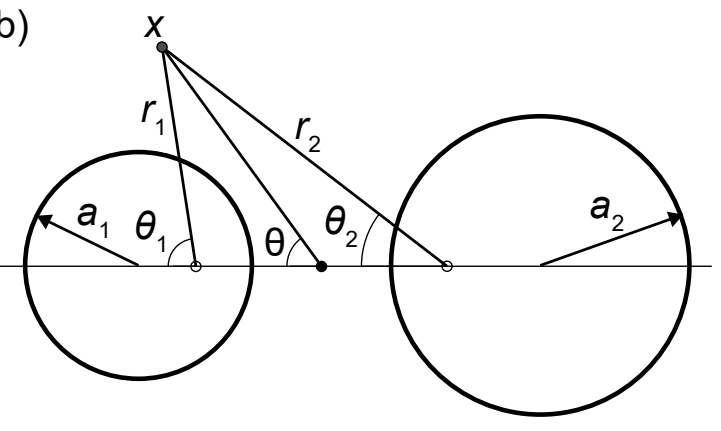

Figure 5: Schematic diagram of geometric parameters in the bispherical coordinate system: (a) $a_{1}$ and $a_{2}$ are the radii of sphere 1 and sphere 2; $a$ is half the separation between the two foci; $s$ is the surface-to-surface separation, and $r$ is the center-to-center separation; $c_{1}$ and $d_{1}$ are inverse-point separations with respect to sphere $1\left(d_{1} c_{1}=a_{1}^{2}\right)$, and $c_{2}$ and $d_{2}$ are inverse-point separations with respect to sphere $2\left(d_{2} c_{2}=a_{2}^{2}\right)$. (b) A position of an arbitrary point $x$ can be described in terms of $\eta \equiv-\ln \left(r_{1} / r_{2}\right), \xi \equiv \theta_{1}-\theta_{2}$, and the azimuthal angle $\varphi$ about the axis that joins the centers of the spheres, where $r_{1}$ and $r_{2}$ are the distances of the point from the two foci and $\theta$ is the angular position of the point relative to the origin (midpoint of the interfocal separation).

The above model for calculating the force requires a determination of the potential in the five regions specified in Figure 6. These potentials in bispherical coordinate system are given by:

$$
\Phi_{i}=\sqrt{\cosh \eta-\cos \xi} \sum_{n=0}^{\infty} \sum_{j=1}^{4} e^{\left(n+\frac{1}{2}\right) \epsilon_{i j}\left(\eta-\eta_{j}\right)} P_{n}(\cos \xi) \Phi_{j, n}, \quad i=1, \ldots, 5
$$




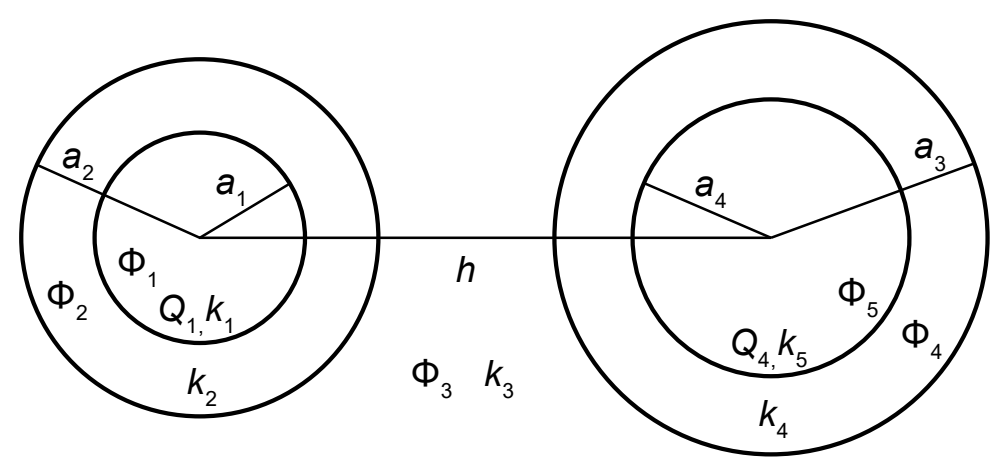

Figure 6: Geometric representation of the 'four-layer' formalism, where $a_{1}$ and $a_{4}$ are the radii of the spheres, $a_{2}$ and $a_{3}$ are the radii of vacuum layers; $\Phi_{1}$ and $\Phi_{5}$ are the potentials inside spheres, $\Phi_{2}$ and $\Phi_{4}$ are the potentials inside the vacuum layers, $\Phi_{3}$ is the potential in the medium, $k_{1}$ and $k_{5}$ are the sphere permittivities (dielectric constants), $k_{3}$ is the dielectric constant of the medium, and $k_{2}=k_{4}=1$, corresponding to the vacuum permittivity. Note that the thickness of the vacuum layers has been made visually finite for clarity.

where

$$
\epsilon_{i j}=-1, \quad j \geq i, \quad i, j=1, \ldots, 5, \quad \epsilon_{i j}=-\epsilon_{i j}=-1, \quad i \neq j
$$

and

$$
\Phi_{i, n}=2 \pi a K \int_{0}^{\pi} \frac{\sigma_{i}\left(\cos \xi^{\prime}\right) \sin \xi^{\prime} d \xi^{\prime}}{\left(\cosh \eta_{i}-\cos \xi^{\prime}\right)^{\frac{3}{2}}} P_{n}\left(\cos \xi^{\prime}\right), \quad i=1, \ldots, 4
$$

or

$$
\sigma_{i}=\left(\cosh \eta_{i}-\cos \xi\right)^{\frac{3}{2}} \sum_{n=0}^{\infty} \frac{2 n+1}{4 \pi a K} \Phi_{i, n} P_{n}(\cos \xi), \quad i=1, \ldots, 4 .
$$

The boundary conditions for uniformly charged spheres are given by $\sigma_{2, f}=\sigma_{3, f}=0$

$$
\begin{aligned}
& \frac{\sigma_{i, f}}{\left(\frac{1}{4 \pi K}\right) \sqrt{\cosh \eta_{i}-\cos \xi}}=\left.\frac{\sqrt{\cosh \eta_{i}-\cos \xi}}{-a}\left(k_{i} \partial_{\eta} \Phi_{i}-k_{i+1} \partial_{\eta} \Phi_{i+1}\right)\right|_{\eta=\eta_{i}} \\
& =\left.\frac{\sqrt{\cosh \eta_{i}-\cos \xi}}{a} \sum_{l=i}^{i+1} \epsilon_{l, i} k_{l} \partial_{\eta} \Phi_{l}\right|_{\eta=\eta_{i}}, \quad i=1, \ldots, 4 .
\end{aligned}
$$

Substituting Equation 11 in Equation 14 gives 


$$
\begin{aligned}
& \frac{a \sigma_{i, f}}{\left(\frac{1}{4 \pi K}\right) \sqrt{\cosh \eta_{i}-\cos \xi}}=\sum_{l=i}^{i+1} \epsilon_{l, i} k_{l} \sum_{n=0}^{\infty} \sum_{j=1}^{4} P_{n}(\cos \xi) \\
& \times\left[\frac{1}{2} \sinh \eta_{i}+\left(\cosh \eta_{i}-\cos \xi\right)\left(n+\frac{1}{2}\right) \epsilon_{l j}\right] e^{\left(n+\frac{1}{2}\right) \epsilon_{l j}\left(\eta_{i}-\eta_{j}\right)} \Phi_{j, n}, \quad i=1, \ldots, 4 .
\end{aligned}
$$

Multiplying above equation by Legendre polynomials, $P_{n}(\cos \xi) \sin \xi d \xi$ and integrating over a unit sphere leads to $\left(\eta_{1}=\eta_{2}=\left|\eta_{1}\right|>0 ; \eta_{3}=\eta_{4}=-\left|\eta_{4}\right|<0\right)$ :

$$
\begin{aligned}
& \frac{\sqrt{2} a}{\left(\frac{1}{4 \pi K}\right)} \sigma_{i, f} e^{-\left(n+\frac{1}{2}\right)\left|\eta_{i}\right|}=\sum_{l=i}^{i+1} \sum_{j=1}^{4} \epsilon_{l, i} k_{l}\left[\frac{1}{2} \sinh \eta_{i} e^{\left(n+\frac{1}{2}\right) \epsilon_{l j}\left(\eta_{i}-\eta_{j}\right)} \Phi_{j, n}+\right. \\
& +\left(n+\frac{1}{2}\right) \epsilon_{l, j} \cosh \eta_{i} e^{\left(n+\frac{1}{2}\right) \epsilon_{l j}\left(\eta_{i}-\eta_{j}\right)} \Phi_{j, n}-\frac{n}{2} \epsilon_{l, j} e^{\left(n-\frac{1}{2}\right) \epsilon_{l j}\left(\eta_{i}-\eta_{j}\right)} \Phi_{j, n-1}- \\
& \left.-\frac{n+1}{2} \epsilon_{l, j} e^{\left(n+\frac{3}{2}\right) \epsilon_{l j}\left(\eta_{i}-\eta_{j}\right)} \Phi_{j, n+1}\right], \quad i=1, \ldots, 4 .
\end{aligned}
$$

The above four boundary conditions can be reduced to two by using the following identities (in the same limit: $\eta_{2} \rightarrow \eta_{1}$ and $\eta_{3} \rightarrow \eta_{4}$ )

$$
\begin{gathered}
\Phi_{1, n}=\sqrt{2} \frac{4 \pi a K \sigma_{1, f}}{k_{1}} \frac{e^{-\left(n+\frac{1}{2}\right) \eta_{1}}}{\sinh \eta_{1}}-\frac{1-\frac{1}{k_{1}}}{1-\frac{1}{k_{3}}} \Phi_{2, n} n, \\
\Phi_{4, n} n=\sqrt{2} \frac{4 \pi a K \sigma_{4, f}}{k_{5}} \frac{e^{-\left(n+\frac{1}{2}\right)\left(-\eta_{4}\right)}}{\sinh \left(-\eta_{4}\right)}-\frac{1-\frac{1}{k_{5}}}{1-\frac{1}{k_{3}}} \Phi_{3, n} .
\end{gathered}
$$

It is then easy to see that the addition of equations corresponding to $i=1$ and $i=2$ from Equation 16 will lead to: 


$$
\begin{aligned}
& \frac{\sqrt{2} a \sigma_{1, f} e^{-\left(n+\frac{1}{2}\right) \eta_{1}}}{\epsilon_{0}} \\
& =\left(\left[\left(n+\frac{1}{2}\right) W_{12, n} \cosh \eta_{1}-\frac{n}{2} W_{12, n-1}-\frac{n+1}{2} W_{12, n+1}\right]\right. \\
& \left.\quad \times\left(k_{3}+k_{1}\right)+\frac{\sinh \eta_{1}}{2}\left(k_{3}-k_{1}\right) W_{12, n}\right) \\
& \quad+\left(-\left[\left(n+\frac{1}{2}\right) W_{34, n} f_{n} \cosh \eta_{1}-\frac{n}{2} W_{34, n-1} f_{n-1}-\frac{n+1}{2} W_{34, n+1} f_{n+1}\right]\right. \\
& \left.\quad \times\left(k_{3}-k_{1}\right)+W_{34, n} f_{n} \frac{\sinh \eta_{1}}{2}\left(k_{3}-k_{1}\right)\right)
\end{aligned}
$$

where

$$
\begin{aligned}
W_{12, n} & =\Phi_{1, n}+\Phi_{2, n} \\
\Phi_{1, n} & =\frac{k_{3}\left(k_{1}-1\right)}{k_{1}-k_{3}} W_{12, n}+\frac{k_{3}-1}{k_{3}-k_{1}} \sqrt{2} 4 \pi K \sigma_{1, f} a_{1} e^{-\left(n+\frac{1}{2}\right) \eta_{1}}, \\
W_{34, n} & =\Phi_{3, n}+\Phi_{4, n} \\
\Phi_{4, n} & =\frac{k_{3}\left(k_{5}-1\right)}{k_{5}-k_{3}} W_{34, n}+\frac{k_{3}-1}{k_{3}-k_{5}} \sqrt{2} 4 \pi K \sigma_{4, f} a_{4} e^{\left(n+\frac{1}{2}\right) \eta_{4}},
\end{aligned}
$$

and

$$
f_{n}=e^{-\left(n+\frac{1}{2}\right)\left(\eta_{1}-\eta_{4}\right)}
$$

Similarly, an addition of equations corresponding to $i=3$ and $i=4$ from Equation 16 will lead to: 


$$
\begin{aligned}
& \frac{\sqrt{2} a \sigma_{4, f} e^{\left(n+\frac{1}{2}\right) \eta_{4}}}{\epsilon_{0}} \\
& =\left(\left[\left(n+\frac{1}{2}\right) W_{34, n} \cosh \eta_{4}-\frac{n}{2} W_{34, n-1}-\frac{n+1}{2} W_{34, n+1}\right]\right. \\
& \left.\quad \times\left(k_{3}+k_{5}\right)+W_{34, n} \frac{\sinh \left(-\eta_{4}\right)}{2}\left(k_{3}-k_{5}\right)\right) \\
& \quad+\left(-\left[\left(n+\frac{1}{2}\right) W_{12, n} f_{n} \cosh \eta_{4}-\frac{n}{2} W_{12, n-1} f_{n-1}-\frac{n+1}{2} W_{12, n+1} f_{n+1}\right]\right. \\
& \left.\quad \times\left(k_{3}-k_{5}\right)+W_{12, n} f_{n} \frac{\sinh \left(-\eta_{4}\right)}{2}\left(k_{3}-k_{5}\right)\right) .
\end{aligned}
$$

Both models presented here produce exactly the same value for the electrostatic force, and therefore they can be used interchangeably.

\section{Appendix B: Effective dipole moment of a polarized sphere}

For the example addressed in Figures 1 and 2, where a neutral dielectric sphere interacts with a charged planar surface, the sphere, under the influence of an electric field generated by the surface, becomes polarized, which leads to the appearance of a dipolar distribution of positive and negative bound charge on the surface of the sphere such that:

$$
\int d Q_{\text {bound }}=\int d Q_{\text {bound }+}+\int d Q_{\text {bound- }}=0 .
$$

The averages of the position vectors $r_{\text {bound+ }}$ and $r_{\text {bound- }}$ of the bound charge elements $d Q_{\text {bound+ }}$ and $d Q_{\text {bound-, }}$, respectively, are given by

$$
\left\langle\mathbf{r}_{\text {bound }+}\right\rangle \equiv \frac{\int \mathbf{r}_{\text {bound }+} d Q_{\text {bound }+}}{\int d Q_{\text {bound }+}}=\frac{\int \mathbf{r}_{\text {bound }+} d Q_{\text {bound }+}}{\delta Q}
$$

and 


$$
\left\langle\mathbf{r}_{\text {bound }-}\right\rangle \equiv \frac{\int \mathbf{r}_{\text {bound }-} d Q_{\text {bound- }}}{\int d Q_{\text {bound- }}}=\frac{\int \mathbf{r}_{\text {bound }-} d Q_{\text {bound }-}}{\delta Q},
$$

where

$$
\delta Q \equiv \int d Q_{\text {bound }+}=-\int d Q_{\text {bound- }} \therefore \int d Q_{\text {bound- }}=-\delta Q
$$

By definition, the effective dipole moment on the neutral polarized sphere can be written as:

$$
\begin{aligned}
\mathbf{p}_{\text {eff }} \equiv \delta Q\left[\left\langle\mathbf{r}_{\text {bound }+}\right\rangle-\left\langle\mathbf{r}_{\text {bound }-}\right\rangle\right] & =\int \mathbf{r}_{\text {bound }+} d Q_{\text {bound }+}+\int \mathbf{r}_{\text {bound }-} d Q_{\text {bound }-} \\
& =\int \mathbf{r}_{\text {bound }} d Q_{\text {bound }} .
\end{aligned}
$$

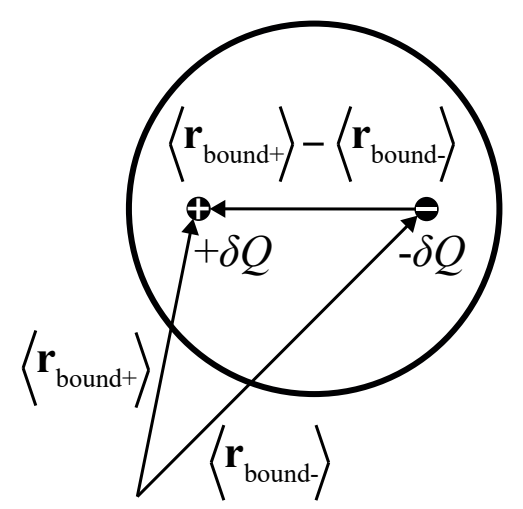

Figure 7: Geometric representation of the various quantities relevant to the calculation of the effective dipole moment of a polarized sphere.

With the origin of the spherical coordinate system at the center of the sphere, positions at the surface of the sphere are as follows: $r=a_{s}$ (sphere radius), $\theta \in\{0, \pi\}$ and $\varphi \in\{0,2 \pi\}$. For a bound charge element, $d Q_{\text {bound }}=\sigma_{\text {bound }} d S$, where $\sigma_{\text {bound }}$ is the surface bound charge density and $d S=a_{s}^{2} \sin \theta d \theta d \varphi$ is a surface element, an effective dipole moment on the polarized sphere can be derived as follows 


$$
\begin{aligned}
\mathbf{p}_{\mathrm{eff}} & =\int r_{\text {bound }} d Q_{\text {bound }} \\
& =a_{s}^{3}\left[\int_{\varphi=0}^{\varphi=2 \pi} \int_{\theta=0}^{\theta=\pi} \sigma_{\text {bound }}(\theta, \varphi) \sin \theta \cos \theta d \theta d \varphi \hat{\mathbf{k}}\right. \\
& +\int_{\varphi=0}^{\varphi=2 \pi} \int_{\theta=0}^{\theta=\pi} \sigma_{\text {bound }}(\theta, \varphi) \sin ^{2} d \theta \cos \varphi d \varphi \hat{\mathbf{i}} \\
& \left.+\int_{\varphi=2 \pi} \int_{\theta=\pi}^{\theta=\pi} \sigma_{\text {bound }}(\theta, \varphi) \sin ^{2} d \theta \cos \varphi d \varphi \hat{\mathbf{j}}\right]
\end{aligned}
$$

The azimuthal symmetry inherent in this two-body problem allows a cancellation of the dependence of surface-bound charge density on the azimuthal angle $\varphi$, such that the second and third terms inside the brackets in Equation 28 become zero and the expression for the effective dipole moment can be simplified to

$$
\begin{aligned}
\mathbf{p}_{\text {eff }} & =a_{s}^{3} \int_{\theta=0}^{\theta=\pi} \sigma_{\text {bound }}(\theta) \sin \theta \cos \theta d \theta \int_{\varphi=0}^{\varphi=2 \pi} d \varphi \hat{\mathbf{k}} \\
& =2 \pi a_{s}^{3} \int_{\theta=0}^{\theta=\pi} \sigma_{\text {bound }}(\theta) \sin \theta \cos \theta d \theta \hat{\mathbf{k}} .
\end{aligned}
$$

Accordingly, the effective polarization (dipole moment per unit volume) can then be defined as

$$
\mathbf{P}_{\mathrm{eff}} \equiv \frac{3}{4} \frac{\mathbf{p}_{\mathrm{eff}}}{\pi a_{s}^{3}}=\frac{3}{2} \int_{\theta=0}^{\theta=\pi} \sigma_{\text {bound }}(\theta) \sin \theta \cos \theta d \theta \hat{\mathbf{k}}
$$




\section{Appendix C: Large sphere approximation to an infinite planar surface}

Although a solution to the dielectric particle - planar surface problem has recently been presented, ${ }^{23}$ calculations show that the solution is not stable if the planar surface is assigned a charge. In order to treat the latter situation, it is shown next that, providing the ratio of their radii is sufficiently large, the particle - planar surface geometry can be approximated by a small sphere interacting with a much larger, charged sphere.

The relative geometry of a system consisting of two spherical objects is dependent upon the relationship between each of the constituent length quantities, namely the radii of the particles and their surface-to-surface separation. For example, if the separation between two spheres is much larger than their radii, the system approaches the geometric limit of two point particles. A quantitative description of this scenario has recently been proposed in the form of a general geometric representation based on the bispherical coordinate system. ${ }^{43}$ The formalism introduces a parameter, $s^{*}=s / 2 a$, where $s$ is the surface-to-surface separation and $2 a$ is the distance between the two inverse points in bispherical coordinates. The parameter approaches the limit of $s^{*}=1$ when the radii of both spheres are much smaller than $s$, i.e. in the limit of two point particles, and approaches the limit $s^{*}=0$ when the radii of both spheres are much larger than $s$. The latter limit also applies to two planar surfaces, which can be regarded as two spheres with infinite radii (Lie sphere geometry). Intermediate cases, for example, a plane - sphere or a point charge - sphere geometries, correspond to values of $s^{*}$ that lie 0 and 1 .

For the geometric arrangement described in Figures 1 and 2, namely a nano-diamond plane and a $50 \mathrm{~nm}$-diameter alumina sphere separated by $s=25 \mathrm{~nm}$, the parameter $s^{*}$ is equal to 0.2887 . In the calculations undertaken here, the nano-diamond plane has been approximated by a large sphere with a diameter a factor of $3 \times 10^{2}$ greater than that of the silica particle; hence the system's geometry is characterized by a value of $s^{*}=0.2896$, 
(b)
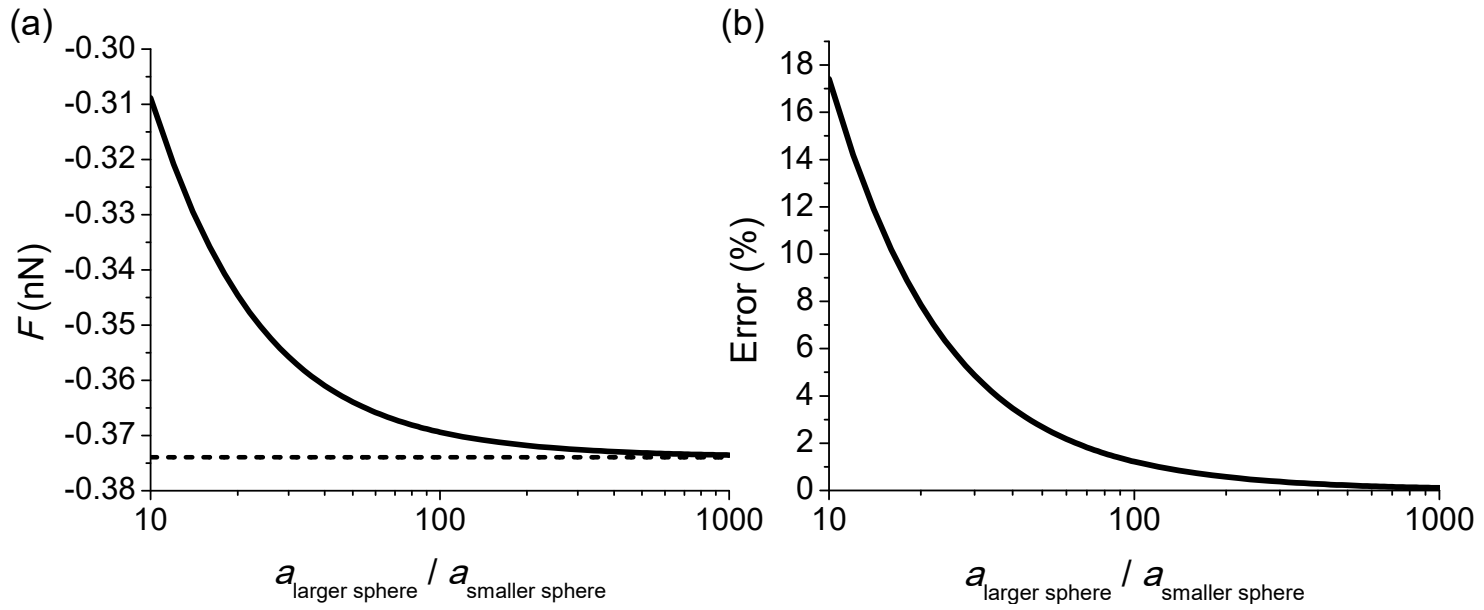

Figure 8: (a) Calculated electrostatic force $(\mathrm{nN})$ as function of the radius ratio between a large, neutral sphere (to mimic an infinite planar surface) and a smaller charged sphere with fixed radius of $25 \mathrm{~nm}$ and charge density $\sigma_{\text {sphere }}= \pm 1 \mathrm{e} \cdot \mathrm{nm}^{-2}$. The dashed line denotes the force obtained by repeating the calculation, but using a solution that can treat an infinite planar surface. ${ }^{23}$ (b) Percentage error between the forces given in (a) as function of the radius ratio.

Figure 8 shows that once the sphere mimicking a surface becomes sufficiently large, the difference between the approximate force and the actual force (obtained by considering an infinite planar surface) becomes negligible. Hence, this approximation provides a mechanism for treating infinite dielectric surfaces that also carry a charge.

In general, the larger the ratio between the radii of the spheres becomes, the greater the number of terms required in the multipole expansion to achieve convergence of the calculated force. For example, in the case of Figure 8, when this ratio is equal to 1000, a minimum of 4000 terms are needed to obtain a force that is converged to five significant figures. A comprehensive discussion of how the number of terms depends not only on the radius ratio, 
but also on other parameters of the system, such as charge ratio, dielectric constant and separation distance, is presented elsewhere. ${ }^{44}$

\section{Acknowledgement}

EBL is supported by a PhD scholarship from the Brazilian Government's Science Without Borders Programme (CAPES: 0702/13-7). EB acknowledges an ERC Consolidator Grant and an EPSRC Impact Acceleration Account.

\section{References}

(1) Bishop, K. J.; Wilmer, C. E.; Soh, S.; Grzybowski, B. A. Nanoscale forces and their uses in self-assembly. Small 2009, 5, 1600-1630.

(2) Caruso, F.; Lichtenfeld, H.; Giersig, M.; Möhwald, H. Electrostatic self-assembly of silica nanoparticle-polyelectrolyte multilayers on polystyrene latex particles. J. Am. Chem. Soc. 1998, 120, 8523-8524.

(3) Smoukov, S. K.; Bishop, K. J.; Kowalczyk, B.; Kalsin, A. M.; Grzybowski, B. A. Electrostatically "patchy" coatings via cooperative adsorption of charged nanoparticles. J. Am. Chem. Soc. 2007, 129, 15623-15630.

(4) Walker, D. A.; Kowalczyk, B.; de La Cruz, M. O.; Grzybowski, B. A. Electrostatics at the nanoscale. Nanoscale 2011, 3, 1316-1344.

(5) Lee, H.; You, S.; Pikhitsa, P. V.; Kim, J.; Kwon, S.; Woo, C. G.; Choi, M. Threedimensional assembly of nanoparticles from charged aerosols. Nano Lett. 2010, 11, $119-124$.

(6) Fudouzi, H.; Kobayashi, M.; Shinya, N. Site-controlled deposition of microsized particles using an electrostatic assembly. Adv. Mater. 2002, 14, 1649-1652. 
(7) Tettey, K. E.; Yee, M. Q.; Lee, D. Layer-by-layer assembly of charged particles in nonpolar media. Langmuir 2010, 26, 9974-9980.

(8) Grzybowski, B. A.; Winkleman, A.; Wiles, J. A.; Brumer, Y.; Whitesides, G. M. Electrostatic self-assembly of macroscopic crystals using contact electrification. Nat. Mater. 2003, 2, 241-245.

(9) Decher, G. Fuzzy nanoassemblies: Toward layered polymeric multicomposites. Science 1997, 277, 1232-1237.

(10) Borges, J. a.; Mano, J. a. F. Molecular interactions driving the layer-by-layer assembly of multilayers. Chem. Rev. 2014, 114, 8883-8942.

(11) Tien, J.; Terfort, A.; Whitesides, G. M. Microfabrication through electrostatic selfassembly. Langmuir 1997, 13, 5349-5355.

(12) Atkins, P.; de Paula, J. Atkins' physical chemistry, 9th ed.; W. H. Freeman, 2010.

(13) Israelachvili, J. N. Intermolecular and surface forces; Academic press, 2011.

(14) Bichoutskaia, E.; Boatwright, A. L.; Khachatourian, A.; Stace, A. J. Electrostatic analysis of the interactions between charged particles of dielectric materials. J. Chem. Phys. 2010, 133, 024105.

(15) Stace, A. J.; Boatwright, A. L.; Khachatourian, A.; Bichoutskaia, E. Why like-charged particles of dielectric materials can be attracted to one another. J. Colloid Interface Sci. 2011, 354, 417-420.

(16) Hyeon, T. Chemical synthesis of magnetic nanoparticles. Chem. Commun. 2003, 927934.

(17) Yin, Y.; Alivisatos, A. P. Colloidal nanocrystal synthesis and the organic-inorganic interface. Nature 2005, 437, 664-670. 
(18) Murray, C. B.; Kagan, C.; Bawendi, M. Synthesis and characterization of monodisperse nanocrystals and close-packed nanocrystal assemblies. Annu. Rev. Mater. Sci. 2000, $30,545-610$.

(19) Serizawa, T.; Hamada, K.-i.; Kitayama, T.; Fujimoto, N.; Hatada, K.; Akashi, M. Stepwise stereocomplex assembly of stereoregular poly (methyl methacrylate)s on a substrate. J. Am. Chem. Soc. 2000, 122, 1891-1899.

(20) Tang, J.; Li, X.-Y.; Wu, H.; Ren, L.-J.; Zhang, Y.-Q.; Yao, H.-X.; Hu, M.-B.; Wang, W. Tube-graft-sheet nano-objects created by a stepwise self-assembly of polymerpolyoxometalate hybrids. Langmuir 2016, 32, 460-467.

(21) Verveniotis, E.; Kromka, A.; Ledinskỳ, M.; Čermák, J.; Rezek, B. Guided assembly of nanoparticles on electrostatically charged nanocrystalline diamond thin films. Nanoscale Res. Lett. 2011, 6, 1-6.

(22) Liu, S.; Kurth, D. G.; Bredenkötter, B.; Volkmer, D. The structure of self-assembled multilayers with polyoxometalate nanoclusters. J. Am. Chem. Soc. 2002, 124, 1227912287.

(23) Khachatourian, A.; Chan, H.-K.; Stace, A. J.; Bichoutskaia, E. Electrostatic force between a charged sphere and a planar surface: A general solution for dielectric materials. J. Chem. Phys. 2014, 140, 074107.

(24) Derbenev, I. N.; Filippov, A. V.; Stace, A. J.; Besley, E. Electrostatic interactions between charged dielectric particles in an electrolyte solution. J. Chem. Phys. 2016, $145,084103$.

(25) Barros, K.; Luijten, E. Dielectric effects in the self-assembly of binary colloidal aggregates. Phys. Rev. Lett. 2014, 113, 017801. 
(30) Lindell, I. V.; Sten, J. C. E.; Nikoskinen, K. I. Electrostatic image method for the interaction of two dielectric spheres. Radio Sci. 1993, 28, 319-329.

(31) Jackson, J. D. Electrodynamics; Wiley: New York, 1975.

(32) Maxwell, J. C. A treatise on electricity and magnetism; Dover Publications Inc: New York, 1954; Vol. 1.

(33) Landau, L. D.; Lífshíts, E. M. Electrodynamics of continuous media, 2nd ed.; Pergamon Press: Oxford, 1984; Vol. 8.

(34) Wu, H.; Han, M.; Luijten, E. Dielectric effects on the ion distribution near a Janus colloid. Soft Matter 2016, 12, 9575-9584.

(35) Liu, S.; Kurth, D. G.; Möhwald, H.; Volkmer, D. A thin-film electrochromic device based on a polyoxometalate cluster. Adv. Mater. 2002, 14, 225.

(36) Proust, A.; Matt, B.; Villanneau, R.; Guillemot, G.; Gouzerh, P.; Izzet, G. Functionalization and post-functionalization: A step towards polyoxometalate-based materials. Chem. Soc. Rev. 2012, 41, 7605-7622. 
(37) Durstock, M. F.; Rubner, M. F. Dielectric properties of polyelectrolyte multilayers. Langmuir 2001, 17, 7865-7872.

(38) Robertson, J. High dielectric constant oxides. Eur. Phys. J. Appl. Phys. 2004, 28, 265-291.

(39) Xia, Y.; Nguyen, T. D.; Yang, M.; Lee, B.; Santos, A.; Podsiadlo, P.; Tang, Z.; Glotzer, S. C.; Kotov, N. A. Self-assembly of self-limiting monodisperse supraparticles from polydisperse nanoparticles. Nat. Nanotechnol. 2011, 6, 580-587.

(40) Schoeler, B.; Kumaraswamy, G.; Caruso, F. Investigation of the influence of polyelectrolyte charge density on the growth of multilayer thin films prepared by the layer-bylayer technique. Macromolecules 2002, 35, 889-897.

(41) Hoogeveen, N. G.; Cohen Stuart, M. A.; Fleer, G. J.; Böhmer, M. R. Formation and stability of multilayers of polyelectrolytes. Langmuir 1996, 12, 3675-3681.

(42) Serizawa, T.; Kamimura, S.; Akashi, M. Electrostatic adsorption of polystyrene particles with different surface charges onto the surface of an ultrathin polymer film. Colloids Surf., A 2000, 164, 237-245.

(43) Chan, H.-K.; Lindgren, E. B.; Stace, A. J.; Bichoutskaia, E. Frontiers in quantum methods and applications in chemistry and physics; Springer, 2015; pp 29-36.

(44) Lindgren, E. B.; Chan, H.-K.; Stace, A. J.; Besley, E. Progress in the theory of electrostatic interactions between charged particles. Phys. Chem. Chem. Phys. 2016, 18, 5883-5895. 